INPLASY

PROTOCOL

To cite: Zhang et al. Efficacy and safety of moxibustion in the treatment of female stress urinary incontinence: A protocol for systematic review and meta-analysis. Inplasy protocol 2021120052. doi: 10.37766/inplasy2021.12.0052

Received: 10 December 2021

Published: 10 December 2021

Corresponding author:

Yueyu Zhang

434719119@qq.com

Author Affiliation:

Hubei University of Traditional Chinese Medicine.

Support: NATCM：2019XZZXZJ006.

Review Stage at time of this submission: Preliminary searches.

Conflicts of interest:

None declared.

\section{Efficacy and safety of moxibustion in the treatment of female stress urinary incontinence: A protocol for systematic review and meta-analysis}

Zhang Y ; Song A ;Zhou Z ; Jiao Y ;Wei D ;Zhu Q ; Shi Y ; Peng B4; Zhang, $Y$.

Review question / Objective: The purpose of this systematic review is to evaluate whether moxibustion is more effective and safer for female patients with stress urinary incontinence. Condition being studied: Stress urinary incontinence is a common genitourinary system disease in women. When abdominal pressure increases with sneezing, coughing or exercise, urine leaks out of the urethra involuntarily. Moxibustion therapy is a traditional Chinese medicine external treatment method. In recent years, it has been more and more widely used in the treatment of female stress urinary incontinence, but there is no systematic evaluation of the efficacy and safety of moxibustion in the treatment of female stress urinary incontinence.

INPLASY registration number: This protocol was registered with the International Platform of Registered Systematic Review and Meta-Analysis Protocols (INPLASY) on 10 December 2021 and was last updated on 10 December 2021 (registration number INPLASY2021120052).

\section{INTRODUCTION}

Review question / Objective: The purpose of this systematic review is to evaluate whether moxibustion is more effective and safer for female patients with stress urinary incontinence.
Rationale: A systematic review and metaanalysis of evidence-based medicine on clinical randomized controlled trials of moxibustion in the treatment of female stress urinary incontinence.

Condition being studied: Stress urinary incontinence is a common genitourinary 
system disease in women. When abdominal pressure increases with sneezing, coughing or exercise, urine leaks out of the urethra involuntarily. Moxibustion therapy is a traditional Chinese medicine external treatment method. In recent years, it has been more and more widely used in the treatment of female stress urinary incontinence, but there is no systematic evaluation of the efficacy and safety of moxibustion in the treatment of female stress urinary incontinence.

\section{METHODS}

Search strategy: The following databases will be searched from the establishment database to December 2021: PubMed, Embase, Web of Science, Cochrane Library, China National Knowledge Infrastructure (CNKI), Chongqing VIP Chinese Science and Technology Journal Database (VIP), Wanfang Database, China Biomedical Literature Database (CBM). We will also search grey literature from World Health Organization Clinical Trials Registry and Chinese Clinical Trail Registry.

Participant or population: Women who are clearly diagnosed as stress urinary incontinence will be included in the study, regardless of age, race, country, or disease course, as long as they meet the recognized diagnostic standards at home and abroad.

Intervention: Moxibustion therapy and related therapies will be used as interventions in this system review, there are no restrictions on moxibustion methods, acupoints, intervention time and treatment course.

Comparator: The control group compared with moxibustion treatment can be shammoxibustion, placebo, conventional drug treatment, and no treatment.

Study designs to be included: All randomized controlled trials that meet the inclusion criteria of stress urinary incontinence will be included in this study. And exclude non-clinical randomized controlled trials, observational studies, case reports, and animal mechanism studies.

Eligibility criteria: 1 . We will include all clinical randomized controlled trials of moxibustion and related therapies for the treatment of stress urinary incontinence, report in English or Chinese, without regional restrictions.2. All review articles, case reports, conference abstracts, crosssectional studies, and observational studies on stress urinary incontinence will be excluded.3. We will retrieve the full text of all relevant articles. If the full text information is not available, the article will be excluded and the reason for the exclusion will be recorded.4. Articles with statistical errors or incomplete data and no access to the original data will be excluded.

Information sources: We will search eight electronic databases, including PubMed, Embase, Web of Science, Cochrane Library, China Knowledge Network (CNKI), China Science and Technology Journal Database (VIP), Wanfang Database and China Biomedical Literature Database (CBM). We will search the above-mentioned database from its establishment to December 2021, the language is limited to Chinese or English, and the research object is female patients with stress urinary incontinence. In addition, we will also search trial registration platforms, including ClinicalTrials.gov and China Clinical Trials Registration Center (ChiCTR), for ongoing or unpublished trials. Reference lists and citation lists of studies that meet the inclusion criteria will also be searched, as well as related systematic reviews to determine further included studies. When the full text information is not available, we will contact the corresponding author to obtain the original information.

Main outcome(s): The main outcome indicators we used were the effective rate after treatment and the change in urine leakage measured by the 1-hour pad test from the baseline.

Additional outcome(s): 1.The International Consultation on Incontinence 
Questionnaire-Urinary Incontinence (ICIQUI) Short Form score; 2.Self-report assessment of therapeutic effect; 3.Severity of urinary incontinence; 4.A 72hour incontinence episode frequency; 5.Frequency and nature of adverse events.

Data management: The two researchers independently read the titles and abstracts of the obtained literature, read the full text of the literature that meets the inclusion criteria to determine whether they really meet the inclusion criteria, discuss the controversial literature or let the third investigator decide whether to include it. Two investigators independently extracted the data included in the study, including methodological content such as study design, intervention measures and methods, measurement, results, hidden grouping, and blinding. The third reviewer checked the consistency of the data. If the required information is incomplete, we will contact the corresponding author to obtain the required information.

Quality assessment / Risk of bias analysis: Two independent reviewers will use the Cochrane risk of bias tool released in 2019 to assess the risk of bias for all studies that meet the inclusion criteria. There are 7 aspects used to evaluate the risk of bias: (1) random sequence generation method; (2) allocation concealment; (3) blinding of subjects and plan implementers; (4) the blinding method of the result assessor; (5) the completeness of the result data; (6) the selective report of the results; (7) other biases. The assessments for each item be classified as low risk, high risk, and unclear. If there is a lack of information in the study regarding the risk of bias assessment, corresponding authors of the study will be contacted. If there is any difference between the above quality evaluation and data extraction process, it should be discussed and resolved by two independent researchers, or a third reviewer should be consulted.

Strategy of data synthesis: Statistical analysis of data will be operated by STATA14.0 software. Dichotomous data will be represented by risk ratio (RR) and 95\% confidence interval (Cl); continuous data will be represented by mean difference (MD) with $95 \%$ CI. If a different measurement scale is used, we will use the standardized mean difference (SMD) for analysis. The chi-square test will be used to assess the heterogeneity of the included studies. If there is no significant heterogeneity $\left(I^{2}<50 \%\right)$, the fixed effects model is used. If there is large heterogeneity $\left(I^{2} \geq 50 \%\right)$, the random effects model is used.

Subgroup analysis: If significant heterogeneity is found in the systematic review and sufficient data are available, we will perform subgroup analysis on the experimental group and the control group based on the type of moxibustion, the duration of moxibustion, the treatment period, and the outcome measurement method.

Sensitivity analysis: If there is significant heterogeneity in the data, we will conduct a sensitivity analysis based on methodological quality, sample size and the impact of missing data to assess the source of heterogeneity and check the stability of the results. Low-quality studies will be excluded and the meta-analysis will be repeated.

Language: The language is limited to Chinese and English.

Country(ies) involved: China.

Keywords: Moxibustion; Acupuncture Therapy; Stress Urinary Incontinence; Systematic Review; Protocol.

Contributions of each author:

Author 1 - Yueyu Zhang.

Author 2 - Aiqun Song.

Author 3 - Zhongyu Zhou.

Author 4 - Yang Jiao.

Author 5 - Dan Wei.

Author 6 - Qiaochu Zhu.

Author 7 - Yue Shi.

Author 8 - Baoyi Peng.

Author 9 - Yangpu Zhang. 